\title{
ONE-DIMENSIONAL LONG-RANGE DIFFUSION-LIMITED AGGREGATION III - THE LIMIT AGGREGATE
}

\author{
GIDEON AMIR
}

\begin{abstract}
In this paper we study the structure of the limit aggregate $A_{\infty}=\bigcup_{n \geq 0} A_{n}$ of the one-dimensional long range diffusion limited aggregation process defined in AABK09. We show (under some regularity conditions) that for walks with finite third moment $A_{\infty}$ has renewal structure and positive density, while for walks with finite variance the renewal structure no longer exists and $A_{\infty}$ has 0 density. We define a tree structure on the aggregates and show some results on the degrees and number of ends of these random trees. We introduce a new "harmonic competition" model where different colours compete for harmonic measure, and show how the tree structure is related to coexistence in this model.
\end{abstract}

\section{INTRODUCTION}

In AABK09 a new 1-dimensional model of diffusion limited aggregation(DLA), that tries to capture the fractal nature of the celebrated DLA model of T. Witten and L. Sander WS83, is defined and studied. The model, defined rigorously in section 2.1 can be described as follows: Start with an aggregate containing a single particle at 0 , at each stage, let a new particle perform a random walk with long jumps starting "from infinity" until it attempts to jump onto the existing aggregate, at which stage the jump is not performed and the particle is glued (added to the aggregate) in its current position. Thus the process generates a sequence $\{0\}=A_{0} \subset A_{1} \subset \ldots$ of disconnected sets in $\mathbb{Z}$, dubbed the aggregates, with the $n$ 'th aggregate $A_{n}$ having $n+1$ points. In AABK09] and AAK13, we study the relation between the diameter of the aggregates, $D_{n}=\operatorname{diam}\left(A_{n}\right)$ and the step-distribution of the underlying random walk $R$. More precisely, denoting by $\alpha(R):=\sup \left\{a \geq 0: \mathbb{E}\left|R_{1}-R_{0}\right|^{a}<\infty\right\}$ - the highest moment of the walk, it is shown that under some regularity conditions the diameters exhibit several phase transitions as the highest moment of the walk varies. A minimal version of these results is given in the following theorem:

Theorem 0 (AABK09 Theorem 1). Let $R$ be a symmetric random walk with step distribution satisfying $\mathbb{P}\left(\left|R_{1}-R_{0}\right|=k\right)=(c+o(1)) k^{-1-\alpha}$. Let $D_{n}$ be the diameter of the $n$ particle aggregate. Then

- If $\alpha>3$, then $n-1 \leq D_{n} \leq C n+o(n)$ a.s., where $C$ is a constant depending only on the random walk.

- If $2<\alpha \leq 3$, then $D_{n}=n^{\beta+o(1)}$ a.s., where $\beta=\frac{2}{\alpha-1}$.

- If $1<\alpha<2$ then $D_{n}=n^{2+o(1)}$ a.s.

- If $\frac{1}{3}<\alpha<1$ then

$$
n^{\beta+o(1)} \leq D_{n} \leq n^{\beta^{\prime}+o(1)}
$$

Date: October 31, 2009. 


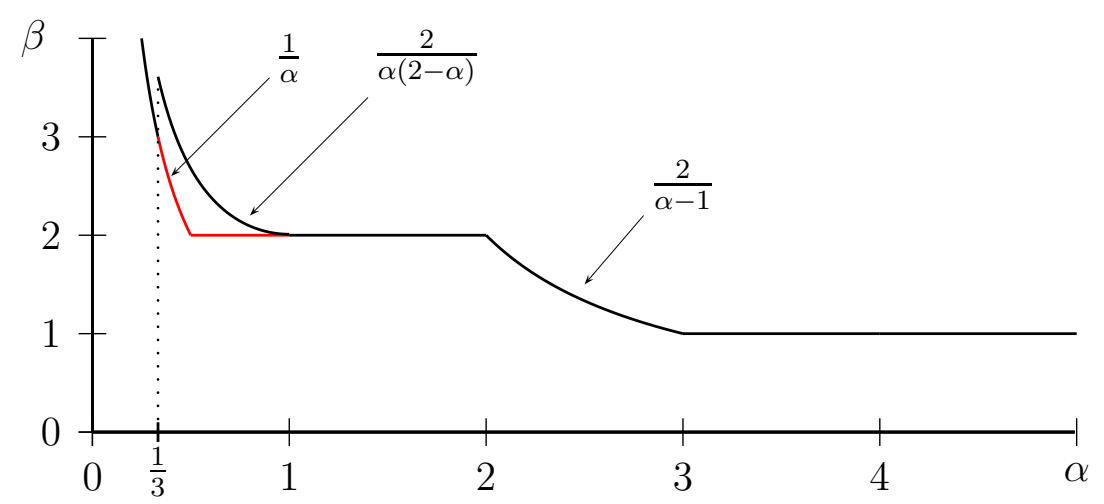

FiguRE 1. From AABK09]: If the random walk $R$ has $\alpha$ finite moments, then the diameter of the resulting $n$-particle aggregate grows as $n^{\beta}$. For $\frac{1}{3}<\alpha<1$ the lower and upper bounds for $\beta$ differ, and the lower bound is conjectured to be correct.

$$
\begin{aligned}
& \text { a.s., where } \beta=\max \left(2, \alpha^{-1}\right) \text { and } \beta^{\prime}=\frac{2}{\alpha(2-\alpha)} \text {. } \\
& \text { - If } 0<\alpha<\frac{1}{3} \text { then } D_{n}=n^{\beta+o(1)} \text { a.s., where } \beta=1 / \alpha \text {. }
\end{aligned}
$$

For explanations concerning the various phase transitions in the theorem, as well as a more thorough introduction to diffusion limited aggregation processes, the reader is referred to the introduction of AABK09.

In this paper, we study the limit aggregate of the 1 dimensional diffusion limited aggregation process, defined simply as $A_{\infty}=\bigcup_{n>0} A_{n}$ - the set of all points eventually added to the aggregates. The natural expectation is that the density of $A_{\infty}$ reflects the growth rate of $D_{n}$, at least to order of magnitude, that is if $D_{n}=n^{\beta+o(1)}$ then

$$
\left|A_{\infty} \cap[-n, n]\right|=n^{1 / \beta+o(1)} .
$$

In this paper, we show that this is indeed the case when $\alpha>2$, and in doing so provide some further detail into the structure of $A_{\infty}$. Our main results are as follows:

Theorem 1. Assume $\mathbb{P}(\xi>t) \leq C t^{-\alpha}$ for any $t$ and some $\alpha>3$. There exists some $B>0$ such that a.s. $A_{\infty}$ has density $B$. Further, $B$ is the limit density of $A_{n}$ :

$$
B=\lim _{m_{1}, m_{2} \rightarrow \infty} \frac{\left|A_{\infty} \cap\left[-m_{1}, m_{2}\right]\right|}{m_{1}+m_{2}}=\lim _{n \rightarrow \infty} \frac{n}{D_{n}} .
$$

Theorem 2. Assume there exist $2<\alpha<3$ and constants $c_{1}, c_{2}>0$ so that $\xi$ satisfies $c_{1} n^{-\alpha} \leq \mathbb{P}(\xi \geq n) \leq c_{2} n^{-\alpha}$ for all $n$ then a.s.

$$
\left|A_{\infty} \cap[-n, n]\right|=n^{\frac{\alpha-1}{2}+o(1)}
$$

In particular, $A_{\infty}$ has 0 density in the sense that $\lim \frac{\left|A_{\infty} \cap[-n, n]\right|}{n}=0$.

To show Theorem 1, we first derive upper bounds on the probability of a random walk passing through a set with $n$ points without hitting it. These bounds, which hold uniformly in the structure of the set, are then used to show that the process has renewal times times at which the subsequent growth of the aggregate is independent of the structure of the aggregate until that time. We show that the set of renewal times dominates a renewal process with positive density, and deduce Theorem 1 as a consequence. 
When $2<\alpha<3$, the strategy of "jumping over" a set to avoid it becomes possible. Using a lower bound on this probability we show the obstacles created by the growing aggregates are not enough to stop new particles from occasionally coming through, and conclude that the renewal structure no longer exists. However, by combining properties of the random walk with simple geometric properties of the aggregates that follow from the diameter growth rates, we are able to show that it is still hard for particles to penetrate deep into the aggregate, and derive the upper bound of Theorem 2. The lower bounds follows from directly from the $2<\alpha \leq 3$ clause of Theorem 0

The case $\alpha<2$ has rather different difficulties and at present we are not ready to speculate on the validity of (11). However, one must make some precautions as in Chapter 7 of AABK09] an example is given of a walk with $\alpha=0$, "the $\mathbb{Z}^{3}$ restricted walk" for which, despite the fact that $D_{n}$ grows faster then any polynomial, $A_{\infty}=\mathbb{Z}$. We do not know if such examples exist for $0<\alpha<2$, as the construction used is somewhat special.

Last, we introduce some additional tree structure onto the aggregates, creating increasing families of random trees which we call the aggregation trees.

To get the aggregation tree $\mathfrak{T}_{n}$ from the aggregate $A_{n}$ and the paths of its particles, we draw an edge between the position at which each particle was stopped when coming from infinity, and the position onto which it attempted to jump. The limit aggregation tree $\mathfrak{T}_{\infty}$ is defined simply as the union of the finite stage trees. Thus $\mathfrak{T}_{\infty}$ combines spatial information (distance) together with the graph structure. Two basic questions on the tree structure are the degree distribution of its vertices and the number of ends in the tree. In section 4 we give a formal definition of these trees, relate them to a competition model where colours compete for harmonic measure and give some partial answers to the above questions.

Acknowledgements. The Author wishes to thank Omer Angel, Itai Benjamini and Gady Kozma for introducing him to diffusion limited aggregation and its 1-dimensional variant and for helpful discussions, and to thank Omer Angel for useful comments on an earlier version of the paper. This research was supported by the Israel Science Foundation (grant No. 1471) and by a Grant from the GIF, the German-Israeli Foundation for Scientific Research and Development.

\section{Preliminaries And Notation}

We will denote a single step of our random walk by $\xi$, and the random walk itself by $R=\left(R_{0}, R_{1}, \ldots\right)$. We will assume through out the paper that our random walk is aperiodic and symmetric. We denote by $\mathbb{P}_{x}$ the probability measure of the random walk started at $x$. We denote by $p_{x, y}$ the probability of the random walk to move from $x$ to $y$ in one step (so $\left.\mathbb{P}(\xi=x)=p_{0, x}\right)$. For a given set $A$, define

$$
p(x, A)=\sum_{a \in A} p_{x, a} .
$$

We denote by $T_{A}$ be the hitting time of $A$, defined as

$$
T_{A}=\min \left\{n>0 \text { s.t. } R_{n} \in A\right\} .
$$

Note that $T_{A}>0$ even if the random walks starts in $A$. For a set $A=\{x\}$ with a single member we also write $T_{x}$. 
We define the hitting measure by

$$
H_{A}(x, a)=\left\{\begin{array}{ll}
\mathbb{P}_{x}\left(R_{T_{A}}=a\right) & x \notin A \\
\delta_{x, a} & x \in A
\end{array} \quad H_{A}( \pm \infty, a)=\lim _{x \rightarrow \pm \infty} H_{A}(x, a)\right.
$$

by [Spi76, T30.1] the limit on the right-hand side exists for any aperiodic random walk. $H_{A}( \pm \infty, \cdot)$ is called the harmonic measure on $A$ from $\pm \infty$. We will set $H_{A}(a)=\frac{1}{2} H_{A}(+\infty, a)+$ $\frac{1}{2} H_{A}(-\infty, a)$ and call it the harmonic measure of $a$ with respect to $A$.

For a subset $A \subset \mathbb{Z}$ we will denote by $\operatorname{diam} A$ the diameter of $A$, namely $\max A-\min A$, by $\bar{A}=[\min A, \max A]$ the minimal interval containing $A$, and by $|A|$ the number of points in $A$. For $x \in \mathbb{Z}$ we will denote by $d(x, A)$ the point-to-set distance, namely $\min _{y \in A}|x-y|$. For convenience we denote by $\mathbb{Z}_{+}$the positive integers including 0 and by $\mathbb{Z}_{-}$the strictly negative integers.

By $C$ and $c$ we denote constants which depend only on the walk $R$ but not on the other parameters involved. The constants hidden in the $o(\cdot)$ notation may also be random. Generally $C$ and $c$ might take different values at different places, even within the same formula. $C$ will usually pertain to constants which are "big enough" and $c$ to constants which are "small enough".

$X \lesssim Y$ denotes that $X<C Y$. By $X \approx Y$ we mean $c X<Y<C X$ (that is, $X \lesssim Y \lesssim X$ ).

In this paper we consider only random walks with finite variance. The following lemma (Lemma 4.2 of [AABK09]) captures some properties of such walks that will be useful for our analysis.

Lemma 3. Let $R$ be a random walk on $\mathbb{Z}$ with steps of mean 0 and variation $\sigma^{2}<\infty$. Then there are $c, C>0$ such that for any $A \subset \mathbb{Z}, A \neq \emptyset$,

(1) If $x>\max A$, then $\lim _{y \rightarrow \infty} \mathbb{P}_{y}\left(T_{x}<T_{A}\right)>c$.

(2) If $d(x, A)$ is large enough then $c<d(x, A) \mathbb{P}_{x}\left(T_{A}<T_{x}\right)<C$.

2.1. DLA as a measure on infinite paths. The purpose of this subsection is to define the DLA generated by a set of random walkers starting "at infinity", in a way that will retain information on the paths of the particles that were used to generate the aggregate. This will allow us to study properties of these paths and relate them to the structure of the aggregates and the limit aggregate, and in particular allow us to define a renewal structure on the aggregation process.

We define the measure $\mathbb{P}_{+\infty}$, depending implicitly on $A$, as follows. This measure is supported on paths $\left\{\gamma_{i}\right\}_{i \leq 0}$, i.e. paths with no beginning but a last step. It is defined as the limit as $y \rightarrow \infty$ of the law of $\left\{R_{T_{A}+i}\right\}_{i \leq 0}$. Informally, $\mathbb{P}_{+\infty}$ is interpreted as the random walk started at $+\infty$, and stopped when it hits $A$. Clearly it is supported on paths in $\mathbb{Z} \backslash A$, except for $R_{0} \in A$. The measure $\mathbb{P}_{-\infty}$ is defined similarly using $y \rightarrow-\infty$. We define the measure $\mathbb{P}_{\infty}=\frac{1}{2}\left(\mathbb{P}_{+\infty}+\mathbb{P}_{-\infty}\right)$.

It was proved in Lemma 2.1 of AABK09] that for recurrent random walks $\mathbb{P}_{+\infty}, \mathbb{P}_{-\infty}$ are probability measures and that for any $x_{0} \in A$ and $x_{-1}, \ldots x_{-n} \in \mathbb{Z} \backslash A$

$$
\mathbb{P}_{ \pm \infty}\left(R_{i}=x_{i} \text { for }-n \leq i \leq 0\right)=\frac{\mathbb{P}_{ \pm \infty}\left(T_{x_{-n}}<T_{A}\right)}{\mathbb{P}_{x_{-n}}\left(T_{A}<T_{x_{-n}}\right)} \prod_{i=-n}^{-1} p_{x_{i}, x_{i+1}}
$$

Let us spend a moment explaining formula (2), as this type of analysis will return later on in the paper. For clarity, write $z=x_{-n}$. Now, in order for the event on the right-hand side to 
happen, the walk must hit $z$ before hitting $A$, which happens with probability $\mathbb{P}_{ \pm \infty}\left(T_{z}<T_{A}\right)$. By the strong Markov property at $T_{z}$, with probability $\mathbb{P}_{z}\left(T_{A}<T_{z}\right)$ the walk will not hit $A$ before its next return to $z$. Thus the expected number of visits to $z$ before $T_{A}$ is

$$
\frac{\mathbb{P}_{ \pm \infty}\left(T_{z}<T_{A}\right)}{\mathbb{P}_{z}\left(T_{A}<T_{z}\right)}
$$

At each of these visits there is probability $\prod_{i=-n}^{-1} p_{x_{i}, x_{i+1}}$ of making the prescribed sequence of jumps ending at $x_{0} \in A$. Since the walk is stopped once such a sequence of jumps is made, the events of making these jumps after the $i$ 'th visit to $z$ are disjoint (for different $i$ 's). Hence summing over these events gives (2).

To define the aggregates of the 1-DLA process, it is enough to use the projection of $\mathbb{P}_{\infty}$ into the last two steps $R_{0}, R_{-1}$ of the random walk, as was done in AABK09. However, some of the events we would like to consider, such as renewal times, will depend on the set of paths used to build the aggregates and not only on the aggregates themselves. We therefore define the 1-DLA process with paths:

Definition 4. Let $R$ be a recurrent random walk on $\mathbb{Z}$. The DLA process with paths with respect to $R$ is a sequence of random tuples $\left\{A_{n}, \Pi_{1}, \ldots \Pi_{n}\right\}_{n \geq 0}$ where $A_{0}=\{0\}, \Pi_{i}$ is chosen according to $\mathbb{P}_{\infty}$ with respect to the set $A_{i-1}$, and $A_{i}=\bar{A}_{i-1} \cup\left\{\Pi_{i}(-1)\right\}$. The sets $A_{0}=\{0\} \subset A_{1} \subset \cdots$ are called the aggregates of the process while $\Pi_{i}$, called the path of the i'th particle, is a backward infinite path on $\mathbb{Z} \backslash A_{i-1}$ ending at $\Pi_{i}(0) \in A_{i-1}$. We set $a_{0}=0$ and $a_{i}=\Pi_{i}(-1)$, thus $A_{n}=\left\{a_{0}, a_{1}, \ldots, a_{n}\right\}$. We call $a_{i}$ the $i$ 'th particle in the aggregate. The limit aggregate of the process, $A_{\infty}$ is defined as the union of all the finite-time aggregates $A_{\infty}=\bigcup_{n \geq 0} A_{n}=\bigcup_{n \geq 0}\left\{a_{n}\right\}$.

We define $\mathcal{F}_{n}$ to be the minimal $\sigma$-field generated by $\Pi_{1}, \ldots \Pi_{n}$. This includes all information about the aggregates $A_{0}, \ldots, A_{n}$.

It is immediate from the definition that the projection of this process onto the sequence $\left\{A_{n}\right\}_{n} \geq 0$ gives back the usual 1-DLA process defined in AABK09.

We say that the $i$ 'th particle started from $+\infty$ if $\lim _{n \rightarrow-\infty} \Pi_{i}(n)=+\infty$, and that the $i$ 'th particle started from $-\infty$ if $\lim _{n \rightarrow-\infty} \Pi_{i}(n)=-\infty$.

Since $\mathbb{P}_{ \pm \infty}\left(\lim _{n \rightarrow-\infty} R_{n}= \pm \infty\right)=1$ with probability 1 every particle has a well defined starting position.

Given the paths constructing the 1-DLA processes, we can now define renewal times:

Definition 5. $n$ is called a weak right renewal time for the $1-D L A$ process if $a_{n}>\max A_{n-1}$, and from time $n$ and on all particles that start from $+\infty$ are added to the aggregate to the right of $a_{n}$.

$n$ is called a strong right renewal time if in addition the paths of all particles that start from $+\infty$ after time $n$ do not hit the half line to the left of $a_{n}$, nor are any such particles glued to a point to the left of $a_{n}$. (i.e. $a_{n}>\max A_{n-1}$ and $\Pi_{i}(j) \geq a_{n}$ for all times $i \geq n$ at which the i'th particle starts from infinity and and for all $j \leq 0$ ).

A symmetric definition holds for left renewal times and particles coming from $-\infty$.

\section{The structure of the limit aggregate}

3.1. Walks with $\alpha>3$. Our starting point for analyzing the limit aggregate for the case $\alpha>3$ is the following theorem from AABK09 bounding diameter of the aggregates $\left\{A_{n}\right\}$ : 
Theorem 6. [AABK09] theorem 4.1] If $\mathbb{E}|\xi|^{3}<\infty$ and $\mathbb{E} \xi=0$ then there is some $C$ so that $\lim \sup \frac{D_{n}}{n}<C$ a.s.

As noted in the introduction, this suggests that the limit aggregate $A_{\infty}$ might have positive density. Theorem 1 state that this is indeed the case. To prove Theorem 1 we study the strong renewal times of the $1-D L A$ process.

Proposition 7. If $\mathbb{P}(\xi>t) \leq C t^{-\alpha}$ for some $\alpha>3$ and all $t>0$, then there exists a constant $c>0$ depending only on $\xi$, such that for any $n$

$$
P\left(n \text { is a strong renewal time } \mid \mathcal{F}_{n-1}\right)>c .
$$

Further, the set of renewal times dominates a renewal process with positive density $c$.

The lower bound given in the proposition is uniform in $\mathcal{F}_{n-1}$ meaning that it holds for almost all possible paths of the first $n-1$ particles, however one should note that the sigma algebra $\mathcal{F}_{n-1}$ does not contain the information what are the renewal times before particle $n$, as to know this one must have information on future paths as well.

To prove the proposition, we will first prove a general lemma on 1-dimensional random walks with finite variance. We will need the following definition:

Definition 8. Let $R$ be a random walk on $Z$ with step distribution $\xi$. We define the (leftoriented) Ladder process of $R$, denoted $L_{R}$, to be the sequence of distinct values attained by the infimum process $\left\{i n f_{l \leq n} R(l)\right\}_{n}$. We denote the step distribution of $L_{R}$ by $L_{\xi}$. Thus $L_{R}$ has strictly negative steps with distribution equal to the hitting measure from 0 of $\mathbb{Z}^{-}$.

Lemma 9. Assume $\mathbb{E}\left(|\xi|^{2}\right)<\infty$. Start the walk $R$ at some $y \geq 0$, and let $\tau=T_{Z_{-}}$. Let $z:=-R(\tau)$ be the overshoot.

(1) There exists a constant $c>0$ such that $\mathbb{P}_{y}(z=1)>c$, uniformly in $y \geq 0(z=1$ means the walk hits the half line at its rightmost point.) In particular for $y=0$ we find $\mathbb{P}\left(L_{\xi}=-1\right)>c$.

(2) There exists a constant $C>0$ s.t. for any $k>0 \mathbb{P}\left(L_{\xi}=-k\right) \leq C \mathbb{P}(\xi \geq k)$.

(3) Assume in addition $\mathbb{P}(\xi>t) \leq C t^{-\alpha}$ for some $\alpha>2$. Then there is some $C>0$ s.t. $\mathbb{P}_{y}(z=k) \leq C k^{1-\alpha}$ for any $k>0$, uniformly in $y \geq 0$.

Note that by translation invariance similar estimates hold for the hitting measure of $(-\infty, x)$ from $y$ for any $x$ and any $y \geq x$.

Proof. (1) By Lemma 3, we know that $\mathbb{P}_{y}\left(T_{0}<T_{Z_{-}}\right) \underset{y \rightarrow \infty}{\longrightarrow} c_{0}>0$, so the requisite bound holds for all large enough $y>y_{0}$. However, the walk can make positive steps as well, so for any $y>0$ there is a positive probability of exceeding $y_{0}$ before hitting the negatives.

(2) Starting the random walk at $0, L_{\xi}=-z$ is the hitting point of $\mathbb{Z}_{-}$. Fix $k>0$, then $\mathbb{P}(z=k)$ is the sum of probabilities of all paths from 0 that hit $\mathbb{Z}_{-}$at $-k$ and terminate there. Partitioning the paths according to the value of $R(\tau-1)$, we get:

$$
\mathbb{P}(z=-k)=\sum_{l \geq 0} \frac{\mathbb{P}_{0}\left(T_{l}<\tau\right) \mathbb{P}(\xi=l+k)}{\mathbb{P}_{l}\left(\tau<T_{l}\right)} .
$$

By Lemma 3, $\mathbb{P}_{0}\left(T_{l}<\tau\right) \leq \frac{C}{l}$, and $\mathbb{P}_{l}\left(\tau<T_{l}\right) \geq \frac{c}{l}$. Together this gives

$$
\mathbb{P}\left(L_{\xi}=-z=-k\right) \leq C \mathbb{P}(\xi \geq k) \text {. }
$$


(3) We partition the paths of the ladder walk from $y$ to $-k$ according to the place from which the ladder walk made the jump to $-k$. Since $L_{R}$ is strictly decreasing, this gives

$$
\begin{aligned}
\mathbb{P}_{y}(z=k) & =\sum_{i=0}^{y} \mathbb{P}\left(L_{R} \text { visits } i\right) \mathbb{P}\left(L_{\xi}=-(i+k)\right) \\
& \leq \sum_{i=0}^{y} \mathbb{P}\left(L_{\xi}=-(i+k)\right) \leq \mathbb{P}\left(L_{\xi} \leq-k\right)<C t^{1-\alpha},
\end{aligned}
$$

where the last inequality follows from the bound of (2) on $L_{\xi}$.

Assume, without loss of generality, that the $n^{\prime} t h$ particles starts from $+\infty$. Let $x_{n}$ be the minimal point in the path of the $n$-th particle and denote $J_{n}=\left|A_{n-1} \cap\left(x_{n}, \infty\right)\right|$, i.e. the number of distinct points in $A_{n-1}$ which the $n^{\prime} t h$ particle has passed before being added to the aggregate. Thus $J_{n}$ measures the amount by which the $n^{\prime} t h$ particle penetrates into the aggregate.

The following lemma is the key ingredient of the proof of Proposition 7 ,

Lemma 10. Assume $\xi$ satisfies $\mathbb{P}(\xi>t) \lesssim t^{-\alpha}$ for some $c>0, \alpha>2$ and all $t>0$. Then there exists a constant $C>0$ s.t.

$$
\mathbb{P}\left(J_{n}>l \mid \mathcal{F}_{n-1}\right) \leq C l^{2-\alpha} \log ^{\alpha-1} l
$$

uniformly in $\mathcal{F}_{n-1}$. In particular the $J_{n}$ 's are stochastically dominated by i.i.d. random variables with the above tail.

Proof. The Lemma is easy consequence of the following statement: There exist positive constants $c, C$ such that for any $m, k>0$ and for any set $A \subset \mathbb{Z}^{+}$with $|A| \geq k m$ and any $y>\max A$,

$$
\mathbb{P}_{y}\left(T_{-}<T_{A}\right)<e^{-c m}+C m k^{2-\alpha} .
$$

Indeed, we may shift $A_{n-1}$ so that it has exactly $l$ non-negative elements, and apply (3) with $A=A_{n-1} \cap \mathbb{Z}^{+}$, with $k=\frac{l}{\text { Clogl }}$ and $m=C \log l$ for a large enough constant $C>0$.

To prove (3), we use the bound

$$
\mathbb{P}_{y}\left(T_{-}<T_{A}\right)<\mathbb{P}_{y}\left(L_{R} \text { avoids } A\right),
$$

since if the ladder process visits $A$, then the corresponding time for the walk $R$ is before $T_{-}$. Henceforth we will only consider the ladder process and its steps and time.

Let $M$ (for many) be the event that there are more then $m$ steps in which $L$ jumps over points in $A$ without landing in $A$. Let $B$ (for big) be the event that $L$ jumps over at least $k$ points of $A$ in a single step. Since $|A| \geq k m$, it is clear that in order to miss $A$, one of $M$ or $B$ must happen. And therefore

$$
\mathbb{P}_{y}\left(T_{-}<T_{A}\right) \leq \mathbb{P}_{y}(M)+P_{y}\left(B \cap M^{c}\right) .
$$

Let $\left\{\rho_{i}\right\}_{i=1}^{I}$ be the sequence of times at which the ladder walk $L_{R}$ passes over or hits points in $A$. Formally, define $\rho_{0}=0$ and define inductively

$$
\rho_{i+1}=\inf \left\{t: A \cap_{7}\left[L(t), L\left(\rho_{i}\right)\right) \neq \emptyset\right\} .
$$


This defines a finite sequence since $A$ is finite. Let $\rho_{I}$ be the time the process passes the last point of $A$. Let $\mathcal{H}_{k}$ be the natural filtration of $L_{R}$. By Lemma 9(1), on the event $i<I$ we have $\mathbb{P}\left(L_{R}\left(\rho_{i+1}\right) \in A \mid \mathcal{H}_{\rho_{i}}\right)>c$. Therefore the probability of avoiding $A$ on at least $m$ jumps is

$$
\mathbb{P}(M) \leq \prod_{i=0}^{m-1} 1-\mathbb{P}\left(L_{R}\left(\rho_{i+1}\right) \in A \mid \mathcal{H}_{\rho_{i}}\right) \leq e^{-c m}
$$

Let $B_{i}$ be the event that $\left|A \cap\left(L_{R}\left(\rho_{i}\right), L_{R}\left(\rho_{i+1}\right)\right)\right|>k$. Then for $B_{i}$ to occur, $L_{R}$ must make a large step. By Lemma 9(3), $\mathbb{P}\left(B_{i} \mid \mathcal{H}_{\rho_{i}}\right)<C k^{2-\alpha}$. we deduce

$$
\mathbb{P}_{y}\left(B_{i} \mid \bigcap_{j=0}^{i-1} B_{j}^{c}\right)<C k^{2-\alpha}
$$

The event $B \cap M^{c}$ implies that at least one of the events $B_{0}, \ldots, B_{I-1}$ occurs, and that $I<m$, therefore

$$
\mathbb{P}_{y}\left(B \cap M^{c}\right) \leq \sum_{i=0}^{I} \mathbb{P}_{y}\left(B_{i} \mid \bigcap_{j=0}^{i-1} B_{j}^{c}\right) \leq m C k^{2-\alpha} .
$$

Together with (44) this yields (3).

Proof of Proposition 7. By symmetry it suffices to prove the proposition for right renewal times. Let $\left\{n_{i}\right\}$ be the sequence of times at which particles start from $+\infty$, and let $\hat{J}_{i}=J_{n_{i}}$ be the amounts by which these particles penetrate the aggregate. By Lemma 10, $\hat{J}_{i}$ are stochastically dominated by i.i.d. random variables: $\hat{J}_{i} \leq Y_{i}$ with

$$
\mathbb{P}\left(Y_{i}>t\right)<C t^{2-\alpha+\varepsilon} \text {. }
$$

Where $\varepsilon>0$ is such that $2-\alpha+\varepsilon<-1$ (or $\varepsilon<\alpha-3$ ). Also, by Lemma 9(1), we can have $\mathbb{P}\left(Y_{i}=0\right)>c>0$.

First, we claim that a sufficient condition for $n_{k}$ to be a strong right renewal time is that $\hat{J}_{i} \leq i-k$ for all $i \geq k$. This follows by induction on $i$ : if at time $n_{i}$ there are at least $i-k$ particles to the right of $a_{n_{k}}$, and $J_{n_{i}}<i-k$ then the path of $a_{n_{i}}$ does not pass $a_{n_{k}}$ and $a_{n_{i}}$ is added to the right of $a_{n_{k}}$. Note that particles arriving from $-\infty$ do not pose a problem here for two reasons. First, these can only increase the number of points to the right of $a_{n_{k}}$ and second, after the first left strong renewal time even that will not occur.

Consider an infinite family of i.i.d. variables $\left\{Y_{i}\right\}_{i \in \mathbb{Z}}$ with distribution as above, coupled with the variables $\left\{J_{i}\right\}_{i \in \mathbb{Z}_{+}}$so that $Y_{i} \geq J_{i}$ for $i \geq 0$. Define

$$
\Upsilon=\left\{n: \forall m \geq n, Y_{m} \leq m-n\right\} .
$$

Equivalently, $\Upsilon$ is the complement of the union of (open) intervals $\bigcup\left(m-Y_{m}, m\right)$. Clearly $\Upsilon$ is a translation invariant renewal process, and by the above, $\Upsilon \cap \mathbb{Z}^{+}$is a subset of the strong right renewal times.

To bound from below the probability that $n$ is a strong renewal time, we find the density of $\Upsilon$. By translation invariance this is

$$
\mathbb{P}(0 \in \Upsilon)=\mathbb{P}\left(\forall m \geq 0, Y_{m}<m\right) \geq \prod_{m \geq 0} c \vee\left(1-(n-m)^{2-\alpha+\varepsilon}\right)>0 .
$$


We are now ready to prove Theorem 1,

Proof of Theorem 1. First, observe that after the first right and left strong renewal times, no particle coming from $-\infty$ affects the growth of the right side of the aggregate. By symmetry it is therefore enough to consider only particles coming from $+\infty$ and show that

$$
\lim _{m \rightarrow \infty} \frac{\left|A_{\infty} \cap[0, m]\right|}{m}=\lim _{n \rightarrow \infty} \frac{n}{D_{n}}
$$

exists and is a.s. constant.

Let $\left\{r_{k}\right\}_{k \geq 1}$ be the sequence of all strong right renewal times. Denote $w_{k}=r_{k+1}-r_{k}$ : the number of particles in the $k^{\prime}$ th renewal interval. Since the growth to the right of the aggregate after time $r_{k}$ does not depend on the history before time $r_{k}$, after the first left renewal time, $w_{k}$ form an i.i.d. sequence. By Proposition 7 and the Renewal Theorem, $\mathbb{E} w_{k}<\infty$, and therefore by the law of large numbers $r_{k}=B_{1} k(1+o(1))$ for some constant $B_{1}$.

Denote $d_{k}=\max A_{r_{k+1}}-\max A_{r_{k}}$ : the diameter of the $k^{\prime}$ th renewal interval. $d_{k}$ also form an i.i.d. sequence. As noted in the proof of Theorem 6, $d_{k}$ is stochastically dominated by $\sum_{i=1}^{w_{k}} Y_{i}$ where $Y_{i}$ are i.i.d. variables with finite expectation, and thus $\mathbb{E} d_{k}<\infty$. We can apply the Renewal Reward Theorem (see e.g. [Dur10]) to conclude that $\sum^{k} d_{i}=B_{2} k(1+o(1))$ for some constant $B_{2} \geq 1$.

The result follows (with $B=B_{1} / B_{2}$ ) for the subsequence where $n$ is a renewal time and $m=\max A_{n}$ at the renewal times. Existence of the limit over all $m$ and $n$ follows by sandwiching $m$ between two renewal points (or $n$ between two renewal times.)

Note that while the above theorem proved that the aggregate has positive density, it also easy to see that under the conditions of the theorem, if $R$ is not a simple random walk, the density of the limit aggregate is not 1 , as holes may happen in any renewal interval.

3.2. Walks with $2<\alpha<3$. When $2<\alpha(R)<3$, the structure of the limit aggregate is quite different from the case $\alpha>3$, as will be seen in theorem 2 and in claim 12 .

Our starting point will be once again the bounds on the diameters $D_{n}$ given in AABK09. The following theorem summarizes the lower bound ([AABK09] theorem 5.1) and the upper bound ([AABK09] theorem 5.3) for our special case:

Theorem 11. Fix $\alpha \in(2,3]$ and let $\beta=\frac{2}{\alpha-1}$. If the random walk is such that $\mathbb{P}(\xi>t) \approx t^{-\alpha}$ and $\mathbb{E} \xi=0$, then a.s. $\max A_{n}=n^{\beta+o(1)}$ and $-\min A_{n}=n^{\beta+o(1)}$.

Note that while the lower bound ([AABK09] theorem 5.1) was stated for $\operatorname{diam}\left(A_{n}\right)$, the proof dealt separately with $\max A_{n}$ and $\min A_{n}$.

We first show that the renewal structure that existed for $\alpha>3$ no longer holds:

Claim 12. If $\mathbb{P}(\xi>t) \approx t^{-\alpha}$ for some $2<\alpha<3$ then there are only finitely many weak renewal times in the $1-D L A$ process.

The main tool for proving the claim, is a lower bound on the probability of hitting a set $A$ while avoiding a set $B$ to its right, given in the next Lemma:

Lemma 13. Assume $P(\xi>t) \geq c t^{-\alpha} \forall t \in \mathbb{R}_{+}$for some constant $c>0$, and $E\left(|\xi|^{2}\right)<\infty$. There is a constant $c>0$, such that for any two finite sets of $A, B$ satisfying $\max A<\min B$ 


$$
\mathbb{P}_{\infty}\left(R\left(T_{A \cup B}-1\right) \in \bar{A}\right) \geq c \cdot(\operatorname{diam}(A)-|A|) \cdot \operatorname{diam}(A \cup B)^{1-\alpha}
$$

Proof. By Lemma 9(1), there is some $c>0$ (independent of $A, B$ ), such that if the random walker is at $\bar{A} \backslash A$, it will hit $A$ before leaving $\bar{A}$ with probability $\geq c$. Therefore it is enough to bound from below the probability that a random walker from infinity hits $\bar{A} \backslash A$ before hitting $\bar{B} \cup A$.

We bound this by decomposing the set of all paths from infinity that hit $\bar{A}$ without hitting $\bar{B}$ according to the place from which the walker jumps to $\bar{A}$, we get (using the same counting argument as in (2))

$$
\begin{gathered}
\mathbb{P}_{\infty}\left(T_{\bar{A} \backslash A}<T_{\bar{B} \cup A}\right)=\sum_{z \notin \bar{A} \cup \bar{B}} \frac{\mathbb{P}_{\infty}\left(T_{z}<T_{\bar{A} \cup \bar{B}}\right) p(z,(\bar{A} \backslash A))}{\mathbb{P}_{z}\left(T_{\bar{A} \cup \bar{B}}<T_{z}\right)} \\
\geq \sum_{z \geq \max (A \cup B)+\operatorname{diam}(A \cup B)} \frac{\mathbb{P}_{\infty}\left(T_{z}<T_{\bar{A} \cup \bar{B}}\right) p(z, \bar{A} \backslash A)}{\mathbb{P}_{z}\left(T_{\bar{A} \cup \bar{B}}<T_{z}\right)} \\
\geq \sum_{z \geq \max (A \cup B)+\operatorname{diam}(A \cup B)} c d(z, A \cup B) p(z, \bar{A} \backslash A) \\
=\sum_{a \in \bar{A} \backslash A}\left(\sum_{z \geq \max (A \cup B)+\operatorname{diam}(A \cup B)} c d(z, A \cup B) p_{z, a}\right) \\
\geq c|\bar{A} \backslash A|(\operatorname{diam}(A \cup B) \mathbb{P}(\xi \geq 2 \cdot \operatorname{diam}(A \cup B)) \\
\geq c \cdot(\operatorname{diam}(A)-|A|) \cdot \operatorname{diam}(A \cup B)^{1-\alpha}
\end{gathered}
$$

The inequality in 5 following by using both parts of Lemma 3 .

Proof of claim 12, : We now use our bounds on the diameter of $A_{n}$ to prove the claim:

Fix some $\varepsilon>0$. Let $n_{0}$ be the (random) minimal $n_{0}$ such that for all $n>n_{0}$

$$
n^{\frac{2}{\alpha-1}-\epsilon} \leq \operatorname{diam}\left(A_{n}\right) \leq n^{\frac{2}{\alpha-1}+\epsilon} .
$$

By Theorem [11, $n^{\frac{2}{\alpha-1}-o(1)} \leq \operatorname{diam}\left(A_{n}\right) \leq n^{\frac{2}{\alpha-1}+o(1)}$ a.s. , so $n_{0}$ is finite a.s.

Fix $n$. Let $E_{k}^{w}(k \geq 0)$ be the event that the particle at time $n+k$ either starts at $-\infty$ or is glued to the right of $A_{n-1}$, and that $(n-1)^{\frac{2}{\alpha-1}-\epsilon} \leq \operatorname{diam}\left(A_{n-1}\right) \leq(n-1)^{\frac{2}{\alpha-1}+\epsilon}$. If $n$ is a weak right renewal time and $n>n_{0}$, then $\bigcap_{k \geq 0} E_{k}^{w}$ must occur. Therefore

$$
\mathbb{P}\left(\mathrm{n} \text { is a weak renewal time and } n>n_{0}\right) \leq \mathbb{P}\left(E_{0}^{w}\right) \prod_{k \geq 1} \mathbb{P}\left(E_{k}^{w} \mid \bigcap_{j=1}^{k-1} E_{j}^{w}\right)
$$

Let $B_{k}=\left\{a_{n}, a_{n+1}, . ., a_{n+k-1}\right\}$. By applying Lemma 13 with respect to the sets $A=A_{n-1}$ and $B=B_{k}$, we get that

$$
\begin{aligned}
& P\left(E_{k}^{w} \mid \bigcap_{j=1}^{k-1} E_{j}^{w}\right) \\
& \quad \leq 1-c \cdot\left(\operatorname{diam}\left(A_{n-1}\right)-|n-1|\right) \cdot \operatorname{diam}\left(A_{n+k-1}\right)^{1-\alpha} \\
& \leq 1-c n^{\frac{2}{\alpha-1}-\epsilon}(n+k)^{\left(\frac{2}{\alpha-1}+\epsilon\right)(1-\alpha)} \\
& \quad \leq 1-c n^{\frac{2}{\alpha-1}}(n+k)^{-2}(n+k)^{-\epsilon \alpha} \\
& 10
\end{aligned}
$$


And therefore

$$
\begin{aligned}
& P\left(\mathrm{n} \text { is a weak renewal time and } n>n_{0}\right) \leq P\left(E_{0}^{w}\right) \prod_{k \geq 1} P\left(E_{k}^{w} \mid \bigcap_{j=1}^{k-1} E_{j}^{w}\right) \\
& \leq \prod_{1 \leq k \leq n} P\left(E_{k}^{w} \mid E_{k-1}^{w}\right) \\
& \leq \prod_{1 \leq k \leq n}\left(1-c n^{\frac{2}{\alpha-1}}(n+k)^{-2}(n+k)^{-\epsilon \alpha}\right) \\
& \leq\left(1-c n^{\frac{2}{\alpha-1}-2-\epsilon \alpha}\right)^{n} \\
& \leq e^{-n \frac{2}{\alpha-1}-1-\epsilon \alpha}
\end{aligned}
$$

Thus for any $2<\alpha<3$ and $\epsilon$ small enough

$$
\sum_{n \geq 1} P\left(\mathrm{n} \text { is a weak renewal time and } n>n_{0}\right)<\infty
$$

So by the Borel-Cantelli lemma there are a.s. only finitely many weak renewal times bigger than $n_{0}$. Since $n_{0}$ is finite a.s., there are a.s. only finitely many weak right renewal times. The case of left renewal times follows by symmetry.

Next we will prove an upper bound on the probability of a random walk hitting $A_{n}$ at time $m>n$, without hitting the points glued to the right or to the left of $A_{n}$. To get this bound we will need some geometric properties of $A_{n}$ (Unlike the lower bound in Lemma 13 which holds for general sets).

The following definition captures the geometric property we will need, which roughly means that the set does not have big gaps between its points, where the gaps are measured on the scale of their position on $\mathbb{Z}$.

Definition 14. A set of positive integers $B=\left\{b_{1}<\cdots<b_{k}\right\}$ is said to be $\varepsilon$-dense if $b_{i+1}<b_{i}^{1+\varepsilon}$ for each $i$. The set is said to be dense in an interval $[n, m]$ if $\{n, m\} \cup(B \cap[n, m])$ is $\varepsilon$-dense. A set $B \subset \mathbb{Z}^{-}$is said to be dense if $-B$ is dense.

We remark that by this definition the empty set, and any singleton, are also considered $\varepsilon$-dense, as they do not contain any gaps.

Lemma 15. Assume $\mathbb{P}(\xi>t)<c t^{-\alpha}$ for some $2<\alpha<3$, and fix $0<\varepsilon<\alpha / 2-1$. Let $B_{+} \subset$ $\mathbb{Z}_{+}$and $B_{-} \subset \mathbb{Z}_{-}$be finite sets. Let $B=B_{-} \cup B_{+}$and set $m=\min \left(\max B_{+}, \min B_{-}\right)$, and $M=\max \left(\max B_{+},-\min B_{-}\right)$. Suppose that $m>2 n, B_{+}$is $\varepsilon$-dense in $\left[n, \max B_{+}\right]$and that $B_{-}$is $\varepsilon$-dense in $\left[\min B_{-},-n\right]$. Then for some $C>0$ depending only on $\varepsilon$ and the walk (and not on $B$ or $n)$, for any $y$ with $|y|>M$

$$
\mathbb{P}_{y}\left(T_{[-n, n]}<T_{B}\right)<C n^{1+\varepsilon} m^{1-\alpha} .
$$

Proof. Define

$$
f(k)= \begin{cases}\sup _{x \geq k} \mathbb{P}_{x}\left(T_{[-n, n]}<T_{B}\right) & k \geq 0, \\ \sup _{x \leq k} \mathbb{P}_{x}\left(T_{[-n, n]}<T_{B}\right) & k<0 .\end{cases}
$$

We will prove by induction on $|k|$ that for a suitable $\gamma$ and any $k \in B$ with $|k|>2 n$,

$$
f(k) \leq \gamma n^{1+\varepsilon} k^{1-\alpha} .
$$


The Lemma follows by considering $k=\max B$ and $k=\min B$.

By symmetry we may assume $k>2 n$. As the base of our induction we will first prove the statement for $2 n<k \leq n^{1+\varepsilon}$. Fix $x>k$ and let $E_{k}$ be the event that the random walker does not hit $k$ before hitting the half line $\left(-\infty, \frac{k}{2}\right)$. Our first task is to bound $\mathbb{P}\left(E_{k}\right)$. Let $z$ be the first point at which the walk hits $(-\infty, k)$. Partitioning according to $z$, we have for any $x \geq k$

$$
\mathbb{P}_{x}\left(E_{k}\right) \leq \sum_{i=\frac{k}{2}}^{k} \mathbb{P}_{x}(z=i) \mathbb{P}_{i}\left(E_{k}\right)+\mathbb{P}_{x}\left(z \leq \frac{k}{2}\right)
$$

By Lemma 9(3), $\mathbb{P}_{x}(z=i) \leq c(k-i)^{1-\alpha}$, and $\mathbb{P}_{x}\left(z \leq \frac{k}{2}\right) \leq c k^{2-\alpha}$. By Lemma $3, \mathbb{P}_{i}\left(E_{k}\right) \leq$ $c \frac{k-i}{k}$ for any $\frac{k}{2} \leq i \leq k$. Combining these bounds gives

$$
\mathbb{P}_{x}\left(E_{k}\right) \leq \sum_{i=k / 2}^{k} \frac{c(k-i)^{2-\alpha}}{k}+c k^{2-\alpha} \leq c k^{2-\alpha}
$$

For $k \in B, k>2 n$ implies $\left\{T_{[-n, n]}<T_{B}\right\} \subset E_{k}$ and thus for any $k \in B$ with $2 n<k \leq n^{1+\varepsilon}$

$$
f(k) \leq \sup _{x \geq k} \mathbb{P}_{x}\left(E_{k}\right) \leq c k^{2-\alpha} \leq c n^{1+\varepsilon} k^{1-\alpha} .
$$

Giving us the basis for our induction $($ for $\gamma>c)$.

It remains to show using induction on $k$ that (9) holds for $k>n^{1+\varepsilon}$.

Let $\tau=T_{(-\infty, k / 2)}$ denote the hitting time of $\left(-\infty, \frac{k}{2}\right)$ by the random walk.

Denote the hitting point by $y=R_{\tau}$ and define the events

$$
Q_{1}=\left\{y<-\frac{k}{4}\right\}, \quad Q_{2}=\left\{|y| \leq \frac{k}{4}\right\}, \quad Q_{3}=\left\{y>\frac{k}{4}\right\} .
$$

We have

$$
\mathbb{P}_{x}\left(T_{[-n, n]}<T_{B}\right)=\sum_{i=1}^{3} \mathbb{P}_{x}\left(Q_{i}, T_{[-n, n]}<T_{B}\right) .
$$

We bound each of the three summands in terms of the value of $f$ at smaller $x$. By Lemma 9 we have $\mathbb{P}_{x}\left(y<-\frac{k}{4}\right)<c k^{2-\alpha}$. By the definition of $f$, this implies

$$
\mathbb{P}_{x}\left(Q_{1}, T_{[-n, n]}<T_{B}\right)<c k^{2-\alpha} f\left(-\frac{k}{4}\right)
$$

Similarly, for any $i \in\left[-\frac{k}{4}, \frac{k}{4}\right]$ we have $\mathbb{P}(y=i)<c k^{1-\alpha}$, and so

$$
\mathbb{P}_{x}\left(Q_{2}, T_{[-n, n]}<T_{B}\right)<c k^{1-\alpha} \sum_{i=-k / 4}^{k / 4} f(i)
$$

To bound the third summand, note that $Q_{3} \cap\left\{T_{[-n, n]}<T_{B}\right\} \subset E_{k}$, and so by (10) and the Markov property at time $\tau$, 


$$
\begin{aligned}
\mathbb{P}_{x}\left(Q_{3}, T_{[-n, n]}<T_{B}\right) & \leq \mathbb{P}_{x}\left(E_{k}\right) \max _{\frac{k}{4}<z<\frac{k}{2}} \mathbb{P}_{z}\left(T_{[-n, n]}<T_{B}\right) \\
& \leq c k^{2-\alpha} f\left(\frac{k}{4}\right) .
\end{aligned}
$$

Combining (11)-(14) gives

$$
\begin{aligned}
f(k) & <c k^{2-\alpha} f\left(-\frac{k}{4}\right)+c k^{2-\alpha} f\left(\frac{k}{4}\right)+c k^{1-\alpha} \sum_{i=-k / 4}^{k / 4} f(i) \\
& <c k^{1-\alpha}\left(k f\left(-\frac{k}{4}\right)+k f\left(\frac{k}{4}\right)+\sum_{i=-k / 4}^{k / 4} f(i)\right) .
\end{aligned}
$$

We will now want to bound $f(i)$ for $i<k / 4$. We will first assume $i>n^{1+\varepsilon}$. At this point we use the fact that $B_{ \pm}$are $\varepsilon$-dense (and therefore $B \cap\left(i^{\frac{1}{1+\varepsilon}}, i\right) \neq \emptyset$.) and that $f$ is by definition decreasing on $\mathbb{Z}^{+}$(and decreasing on $\mathbb{Z}^{-}$). Together with the induction hypothesis these facts imply

$$
f(i) \leq \gamma n^{1+\varepsilon}|i|^{\frac{1-\alpha}{1+\varepsilon}}<\gamma n^{1+\varepsilon}|i|^{1-\alpha+2 \varepsilon} \quad \text { as long as }|i|>n^{1+\varepsilon} .
$$

For $i<n^{1+\varepsilon}$ we will use the trivial bound $f(i) \leq 1$.

Using these bounds in (15) gives

$$
\begin{aligned}
f(k) & <c k^{1-\alpha} 2 n^{1+\varepsilon}+c k^{1-\alpha} \gamma n^{1+\varepsilon}\left(2 k(k / 4)^{1-\alpha+2 \varepsilon}+2 \sum_{i=n^{1+\varepsilon}}^{k / 4} i^{1-\alpha+2 \varepsilon}\right) \\
& <c k^{1-\alpha} 2 n^{1+\varepsilon}+c k^{1-\alpha} \gamma n^{1+\varepsilon}\left(k^{2-\alpha+2 \varepsilon}+n^{(1+\varepsilon)(2-\alpha+2 \varepsilon)}\right) .
\end{aligned}
$$

Here $c$ is some constant depending only on the random walk. To get the claimed bound on $f(k)$ we need this to be less than $\gamma n^{1+\varepsilon} k^{1-\alpha}$. This happens iff

$$
2 c+\gamma c\left(k^{2-\alpha+2 \varepsilon}+n^{(1+\varepsilon)(2-\alpha+2 \varepsilon)}\right)<\gamma .
$$

We can easily find $\gamma, n_{0}$ so that this holds for any $k>n>n_{0}$.

We now have all the pieces to prove Theorem 2

Proof of Theorem 2. For the lower bound on $A_{\infty} \cap[-n, n]$ it is enough to note that by Theorem 0 the first $n^{\frac{\alpha-1}{2}+o(1)}$ points of the aggregate a.s. lie in the interval $[-n, n]$. We are therefor left with showing the upper bound.

Fix $\varepsilon>0$, which we may assume to be small enough. It is enough to prove that there a.s. exists a constant $C$ so that $\left|A_{\infty} \cap[-n, n]\right| \leq C n^{\frac{\alpha-1}{2}+\varepsilon}$ for all $n$. We will first show that the aggregates $A_{m}$ satisfy the geometric property required for Lemma 15 for large enough $m$ :

Lemma 16. For any $\varepsilon>0$ there is some $n_{0}$ s.t. for any $m>n>n_{0}$

(1) $m^{\frac{2}{\alpha-1}-\varepsilon} \leq \max A_{m} \leq m^{\frac{2}{\alpha-1}+\varepsilon}$

(2) $m^{\frac{2}{\alpha-1}-\varepsilon} \leq-\min A_{m} \leq m^{\frac{2}{\alpha-1}+\varepsilon}$

(3) $A_{m}$ is $\varepsilon$-dense in $\left[\min A_{m}, \min A_{n}\right]$ and in $\left[\max A_{n}, \max A_{m}\right]$. 
Proof. The first two clauses are just a restatement of 11, To prove the third clause, observe that if there is no point in $A_{m}$ between $k$ and $k^{1+\varepsilon}$ (or between $-k$ and $-k^{1+\varepsilon}$ ), then the diameter of the aggregates must grow by too much in one step, contradicting the diameter bounds.

We now return to the proof of theorem 2, Fix some $n>n_{0}$. For any $m>n$

$$
\mathbb{P}\left(a_{m+1} \in \overline{A_{n}}\right) \leq \sup _{|x| \geq \max _{a \in A_{m}}|a|} \mathbb{P}_{x}\left(T_{\overline{A_{n}}}<T_{A_{m} \backslash \overline{A_{n}}}\right)
$$

Take $m_{0}=8 n^{1+3 \varepsilon}$. By the above lemma $\max A_{n} \leq n^{\frac{2}{\alpha-1}+\varepsilon},-\min A_{n} \leq n^{\frac{2}{\alpha-1}+\varepsilon}$ and also $\max A_{m} \geq m^{\frac{2}{\alpha-1}-\varepsilon},-\min A_{m} \geq m^{\frac{2}{\alpha-1}-\varepsilon}$. In particular for any $m>m_{0}, \max A_{m} \geq$ $2 \max A_{n},-\min A_{m} \geq-2 \min A_{n}$, and by the third clause of Lemma 16 $A_{m}$ is $\varepsilon$-dense in $\left[\min A_{m}, \min A_{n}\right]$ and in $\left[\max A_{n}, \max A_{m}\right]$

thus we can apply Lemma 15 to get

$$
\begin{gathered}
\sup _{|x| \geq \max _{a \in A m}|a|} \mathbb{P}_{x}\left(T_{\overline{A_{n}}}<T_{A_{m} \backslash \overline{A_{n}}}\right) \leq \\
\leq C n^{\left(\frac{2}{\alpha-1}+\varepsilon\right)(1+\varepsilon)}\left(m^{\frac{2}{\alpha-1}-\varepsilon}\right)^{1-\alpha} \leq C n^{\frac{2}{\alpha-1}+4 \varepsilon} m^{-2+2 \varepsilon}
\end{gathered}
$$

Let $W_{m_{0}, n}$ be the number of points added to $\left[\min A_{n}\right.$, max $\left.A_{n}\right]$ after time $m_{0}$. Then

$$
\mathbb{E}\left(W_{m_{0}, n}\right) \leq \sum_{m \geq m_{0}} C n^{\frac{2}{\alpha-1}+4 \varepsilon} m^{-2+2 \varepsilon} \leq C n^{\frac{2}{\alpha-1}+4 \varepsilon} m_{0}^{2 \varepsilon-1} \leq C n^{\frac{2}{\alpha-1}-1+9 \varepsilon}
$$

Since our bounds are uniform in the history for all $m>m_{0}$, and they are all indicator variables, the variance of their sum is less then the expectation, and by Chebyshev's inequality

$$
\mathbb{P}\left(W_{m_{0}, n}>2 C n^{\frac{2}{\alpha-1}-1+9 \varepsilon}\right) \leq \frac{C}{n^{\frac{2}{\alpha-1}-1+9 \varepsilon}}
$$

and therefore there is some $N_{0}$ such that this does not happen for any $n>N_{0}$. Thus for any $n>N_{0}$ we add at most $m_{0}=8 n^{1+3 \varepsilon}$ points until time $m_{0}$, and $C n^{\frac{2}{\alpha-1}-1+9 \varepsilon}$ after time $m_{0}$. Since $\varepsilon$ was arbitrary, we are done.

\section{The AgGregation tree}

We start by giving the definition of the aggregation trees promised in the introduction

Definition 17. Given a 1-DLA process $\left\{A_{n}, \Pi_{1}, \ldots \Pi_{n}\right\}_{n \geq 0}$ we define the $n^{\prime}$ th aggregation tree $\mathfrak{T}_{n}$ as the graph whose vertices are the points in $A_{n}$, and whose edge set is $\left\{\left(\Pi_{i}(0), \Pi_{i}(-1)\right)\right\}_{i \leq n}$. The limit aggregation tree is defined by $\mathfrak{T}_{\infty}=\bigcup_{n \geq 0} \mathfrak{T}_{n}$. These trees can be given a directed structure by directing each edge from $\Pi_{i}(0)$ to $\Pi_{i}(1)$.

Note that by the definition of the aggregation tree, the probability of adding an edge from a vertex $n \in \mathfrak{T}_{k}$ at time $k+1$ is $\mathbb{P}_{\infty}(R(0)=n)=H_{A_{k}}(n)$ - the harmonic measure of $n$ with respect to $A_{k}$.

We will be interested in two types of properties on $\mathfrak{T}_{\infty}$ - the degrees of its vertices and the number of ends it possesses. 
The number of ends in a tree is the maximal cardinality of the number of vertex-almostdisjoint infinite simple paths in the tree. (Almost disjoint meaning every pair of paths sharing only finitely many vertices).

This can also be thought of in terms of a coexistence of different species in a competition model: Start by choosing $n$ and colouring the points of $\mathfrak{T}_{n}$ with different colours and then grow the aggregation tree using the DLA dynamics, colouring each new point by the colour of the point to which it was glued. A colour is said to survive if its component in $\mathfrak{T}_{\infty}$ is infinite. It is not hard to see that the maximal colours that can coexist is $\geq n$ if and only if the number of ends in $\mathfrak{T}_{\infty}$ is $\geq n$.

The renewal structure for $\alpha>3$ easily implies the number of ends.

Lemma 18. If $\mathbb{P}(\xi>t) \lesssim t^{-\alpha}$ for $\alpha>3$ then a.s. $\mathfrak{T}_{\infty}$ has 2 ends.

Proof. Let $\tau_{i}$ be the right strong renewal times. Any path $P$ in $\mathfrak{T} \infty$ which is not bounded above $\left(\left|P \cap Z_{+}\right|=\infty\right.$ has to include all $a_{\tau_{i}}$ for all large enough $i$. Therefore there cannot be 2 almost disjoint such paths. The renewal times also ensure that there are two disjoint paths one going to $+\infty$ and one to $-\infty$, so $\mathfrak{T}_{\infty}$ has 2 ends.

Remark. To understand the difficulties in finding the number of ends without the renewal structure, we take another look at the competition model. At each stage, the point to which the next particle is glued is distributed according to the harmonic measure on the aggregate. Thus the colour of the next particle is distributed proportional to the harmonic measure of each colour with respect to the aggregate. When a Red particle is added, the harmonic measure the red part of the aggregate increases, while the harmonic measure of all other colours decreases. Thus the colours can be thought of as competing for harmonic measure. For Red to die out, its harmonic measure must decrease so that $\sum_{n \geq 0} H_{A_{n}}(R e d)$ is finite, otherwise red points will occur infinitely often almost surely. The problem is that a small number of points added to the aggregate can make a big difference in the harmonic measure on the aggregate. Consider a random walk $R$ with finite variance. Take the competition process between Red and Blue, and assume that at time $n$ a new red point is added to the right of $A_{n-1}$. The harmonic measure of that point is at least a constant. Therefore even if Red started to die out, one point is enough to increase its harmonic measure to at least a constant.

In light of the above we ask

Question 1 . How many ends does $\mathfrak{T}_{\infty}$ have for walks with $2<\alpha<3$

Two other natural questions which hold in many models of random trees are the following:

Question 2. Is the number of ends in $\mathfrak{T}_{\infty}$ a.s. constant (or infinite) for any random walk $R$ ?

Question 3. Is the number of ends in $\mathfrak{T}_{\infty}$ always in $\{1,2, \infty\} ?$

The difficulty comes from the high dependency structure between particles added at different steps to the aggregate.

We now go on to study some properties of the degrees of the vertices in $\mathfrak{T}_{\infty}$.

The first lemma shows that for walks with finite variance the degrees are not uniformly bounded.

Lemma 19. If $\mathbb{E}\left(|\xi|^{2}\right)<\infty$ then $\sup _{v \in \mathfrak{T}_{\infty}} \operatorname{deg}(v)=\infty$ 
Proof. Every time a particle $a_{k}$ is added to the right of the aggregate, there is a positive probability depending only on $n$ (and not on $A_{k}$ ) that the next $n$ particles will all come from $+\infty$ and glue to $a_{k}$ without ever jumping over $a_{k}$. Since particles glue on the right i.o., we will almost surely have vertices with degree $>n$ for any $n$.

We now show that under general conditions, having a vertex with infinite degree implies $A_{\infty}=\mathbb{Z}:$

Proposition 20. Assume $\mathbb{E}(|\xi|)<\infty$ and for every $k \in \mathbb{Z}$ there exist constants $c_{k}, C_{k}>0$ such that $c_{k}<\frac{p_{x, k}}{p_{x, 0}}<C_{k}$ for all $x \neq 0, k$.

Then for any $n_{0} \in \mathfrak{T}_{\infty} \mathbb{P}\left(\left\{\operatorname{deg}_{\mathfrak{T}_{\infty}}\left(n_{0}\right)=\infty\right\} \cap\left\{n_{1} \notin A_{\infty}\right.\right.$ or $\left.\left.\operatorname{deg}_{\mathfrak{T}_{\infty}}\left(n_{1}\right)<\infty\right\}\right)=0$. And in particular if there a.s. exists some $n_{0} \in \mathfrak{T}_{\infty}$ with infinite degree then a.s. all vertices in $\mathfrak{T}_{\infty}$ have infinite degree and $A_{\infty}=\mathbb{Z}$ a.s. .

Proof. We will use Levy's extension to the Borell-Cantelli Lemma (see e.g. [Wil91], Thm 12.15)

Lemma 21. Let $E_{n}$ be a sequence of events. And let $\mathcal{G}_{n}=\sigma\left\{E_{1}, \ldots, E_{n}\right\}$. Then

$$
\mathbb{P}\left(\left\{\lim \sup E_{i}\right\} \triangle\left\{\sum_{n \geq 0} \mathbb{P}\left(E_{i} \mid \mathcal{G}_{n-1}\right)\right\}\right)=0
$$

Assume the degree of $n_{0}$ in $\mathfrak{T}_{\infty}$ is infinite. Let $t_{o}$ be the time at which it was added to the aggregate. Let $I_{k}$ be the indicator of the event that an edge is connected to $n_{0}$ at step $k+1$. Then $\mathbb{P}\left(I_{k}=1 \mid \mathcal{G}_{k-1}\right)=H_{A_{k}}\left(n_{0}\right)$, and the degree of $n_{0}$ in $\mathfrak{T}_{\infty}$ is just $1+\sum_{k \geq t_{0}} I_{k}$.

By Levy's extension to the Borell-Cantelli $\mathbb{P}\left(\left\{\operatorname{deg}_{\mathfrak{T}_{\infty}}\left(n_{0}\right)=\infty\right\} \triangle\left\{\sum_{k \geq t_{0}} H_{A_{k}}\left(n_{0}\right)=\infty\right\}\right)=$ 0 . Fix any $n_{1} \in \mathbb{Z}$, and let $t_{1}$ be a time at which both $n_{0}$ and $n_{1}$ are in $A_{t_{1}}$. Decomposing paths from $\pm \infty$ to $n_{1}$ by the position from which they jumped, we get,by (2) that for any set $A$ with $n_{0}, n_{1} \in A$

$$
\begin{aligned}
& H_{A}\left(n_{1}\right)=\mathbb{P}_{\infty}\left(R(0)=n_{1}\right)=\sum_{x \notin A} \mathbb{P}_{\infty}\left(R(0)=n_{1}, R(-1)=x\right) \\
& =\sum_{x \notin A} \frac{\mathbb{P}_{\infty}\left(T_{x}<T_{A}\right)}{\mathbb{P}_{x}\left(T_{A}<T_{x}\right)} p_{x, n_{1}} \approx \sum_{x \notin A} \frac{\mathbb{P}_{\infty}\left(T_{x}<T_{A}\right)}{\mathbb{P}_{x}\left(T_{A}<T_{x}\right)} p_{x, n_{0}}=H_{A}\left(n_{0}\right)
\end{aligned}
$$

Where the constants in the $\approx$ come from the condition on the walk (and do not depend on $A)$. We deduce $\mathbb{P}\left(\left\{\operatorname{deg}_{\mathfrak{T}_{\infty}}\left(n_{0}\right)=\infty\right\} \triangle\left\{\sum_{k \geq t_{1}} H_{A_{k}}\left(n_{1}\right)=\infty\right\}\right)=0$ which implies, using again Levy's extension to the Borell-Cantelli Lemma, that $\mathbb{P}\left(\left\{\operatorname{deg}_{\mathfrak{T}_{\infty}}\left(n_{0}\right)=\infty\right\} \triangle\left\{\operatorname{deg}_{\mathfrak{T}_{\infty}}\left(n_{1}\right)=\right.\right.$ $\infty\})=0$.

To show that $\left.\mathbb{P}\left(\operatorname{deg}_{\mathfrak{T}_{\infty}}\left(n_{0}\right)=\infty\right\} \triangle\left\{A_{\infty}=\mathbb{Z}\right\}\right)=0$, we use a similar argument: Fix any $n_{1} \in \mathbb{Z}$. by (2)

$$
\begin{array}{r}
H_{A}\left(n_{0}\right)=\mathbb{P}_{\infty}\left(R(0)=n_{0}\right)=\sum_{x \notin A} \mathbb{P}_{\infty}\left(R(0)=n_{0}, R(-1)=x\right) \\
=\sum_{x \notin A} \frac{\mathbb{P}_{\infty}\left(T_{x}<T_{A}\right)}{\mathbb{P}_{x}\left(T_{A}<T_{x}\right)} p_{x, n_{1}} p_{n_{1}, n_{0}} \geq \sum_{x \notin A} \frac{\mathbb{P}_{\infty}\left(T_{x}<T_{A}\right.}{\mathbb{P}_{x}\left(T_{A}<T_{x}\right)} p_{x, n_{1}} p_{n_{1}, A}=\mathbb{P}_{\infty}\left(R(-1)=n_{1}\right)
\end{array}
$$

We therefore conclude $\mathbb{P}\left(\left\{\operatorname{deg}_{\mathfrak{T}_{\infty}}\left(n_{0}\right)=\infty\right\} \triangle\left\{\sum_{k \geq t_{0}} \mathbb{P}_{\infty}^{A_{k}}\left(R(-1)=n_{1}\right)=\infty\right\}\right)=0$ and by another use of Levy's extension $\mathbb{P}\left(\left\{\operatorname{deg}_{\mathfrak{T}_{\infty}}\left(n_{0}\right)=\infty\right\} \triangle\left\{n_{1} \in A_{\infty}\right\}\right)=0$ 
Remark. (1) Since only finitely many points in $A_{\infty}$ can be close to any point $n_{0}$, with some extra work the condition on the walk can be weakened to $\lim \inf _{|n| \rightarrow \infty} \frac{p_{n, 0}}{p_{n, k}}>c_{k}$ and $\lim \sup _{|n| \rightarrow \infty} \frac{p_{n, 0}}{p_{n, k}}<C_{k}$ for any $k \in \mathbb{Z}$.

(2) A similar theorem holds for transient random walks $(\mathbb{E}(|\xi|)=\infty)$ using the gluing formula for transient walk ([AABK09] section 7)

Using the above we get that for nice walks with finite variance, all degrees in $\mathfrak{T}_{\infty}$ are a.s. finite

Corollary 22. If $\mathbb{P}(\xi>t) \lesssim t^{-\alpha}$ for $\alpha>3$, or $\mathbb{P}(\xi>t) \approx t^{-\alpha}$ for $3>\alpha>2$ then all degrees in $\mathfrak{T}_{\infty}$ are a.s. finite.

Proof. For the $\alpha>3$ this follows from the renewal structure, since after the first left and right renewal times, only points within a renewal interval can connect to points in that interval (with the exception the renewal points themselves which have one connection to the previous interval). For $2<\alpha<3$ Theorem 2 implies $A_{\infty} \neq \mathbb{Z}$ a.s. and therefore by Proposition 20 all degrees in $\mathfrak{T}_{\infty}$ are a.s. finite.

\section{REFERENCES}

[AABK09] Gideon Amir, Omer Angel, Itai Benjamini, and Gady Kozma. One-dimensional long-range diffusion-limited aggregation i. To Appear in Annals of Probability. Preprint at arXiv:0910.4416, 2009.

[AAK13] Gideon Amir, Omer Angel, and Gady Kozma. One-dimensional long-range diffusion limited aggregation ii: the transient case. arXiv preprint arXiv:1306.4654, 2013.

[Dur10] Rick Durrett. Probability: theory and examples. Cambridge university press, 2010.

[Spi76] Frank Spitzer. Principles of random walks. Springer-Verlag, New York, second edition, 1976. Graduate Texts in Mathematics, Vol. 34.

[Wil91] David Williams. Probability with martingales. Cambridge university press, 1991.

[WS83] T. A. Witten and L. M. Sander. Diffusion-limited aggregation. Phys. Rev. B (3), 27(9):5686-5697, 1983.

\section{Gideon Amir}

Department of Mathematics, Bar-Ilan University

Ramat Gan 52900, Israel

GIDI.AMIR@GMAIL.COM 